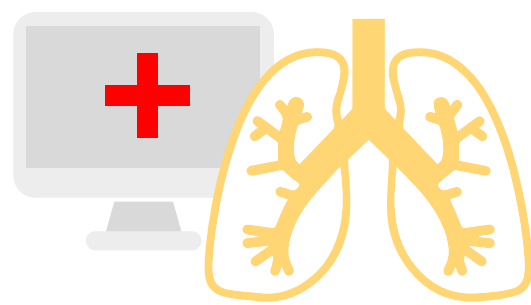

\title{
TECNOLOGIA APLICADA À SAÚDE NA PREVENÇÃO DA PNEUMONIA ASSOCIADA À VENTILAÇÃO MECÂNICA
}

\author{
TECHNOLOGY APPLIED TO HEALTH TO PREVENT \\ PNEUMONIA VENTILATOR-ASSOCIATED
}

\begin{abstract}
Elisana Silva da Rosa
Bacharel em Enfermagem pela Universidade Feevale (2018). Atualmente, é pós-graduando em Terapia Intensiva e Emergência Adulto pela Faculdade de Ciências da Saúde no Hospital Moinhos de Vento. Membro do Grupo de Pesquisa Computação Aplicada da Universidade Feevale em 2017. elisanadarosa@ terra.com.br

\section{Christian Negeliskii}

Doutor em Enfermagem pela UFRGS (2015). Mestre em Enfermagem pela UFRGS (2010). Especialista em Informação Científica e Tecnológica - Fio Cruz (2006) e em UTI Adulto - ULBRA (2001). Atualmente, é gerente de risco assistencial do HNSC (Hospital Nossa Senhora da Conceição) e professor de graduação em Enfermagem e coordenador da pós-graduação em UTI Adulto da Universidade Feevale. Membro do Grupo de Pesquisa Computação Aplicada - Feevale, desde 2016. chnege|@feevale.br
\end{abstract}

\section{Henrique Rick}

Graduando em Ciência da Computação pela Universidade Feevale. Técnico em Informática pelo Instituto de Educação Ivoti (2013). Bolsista do Grupo de Computação Aplicada da Universidade Feevale (2017).

Possui experiência em Infraestrutura de TI. Atualmente, é desenvolvedor ADVPL (Advanced Protheus Language) na Hercosul Alimentos. henriquerick95@hotmail.com

\section{Juliano Varella de Carvalho}

Doutor em Ciência da Computação pela PUCRS (2015). Professor adjunto dos cursos de Computação da Universidade Feevale desde 2003 e coordenador do curso de Ciência da Computação dessa instituição desde 2008. Atuou entre 2001 e 2007 como programador, analista de sistemas e diretor da empresa WZero Soluções para Internet. Sua atuação acadêmica concentra-se nas áreas de Ciência de Dados, Tecnologias para a área da Saúde, MapReduce, Desenvolvimento de Sistemas e Wearable Devices. julianovc@feevale.br

\section{Marta Rosecler Bez}

Doutora em Informática na Educação pela UFRGS. Mestre em Ciência da Computação pela PUC-RS. Professora da Universidade Feevale nos cursos de Computação, Medicina e no Mestrado de Indústria

Criativa. Possui experiência na área de ensino e da saúde permeada por tecnologias. Bolsista de Produtividade em Desenvolvimento Tecnológico e Extensão Inovadora do CNPq - Nível 2 com o projeto Novas Tecnologias Aplicadas ao Ensino na Área da Saúde.martabez@gmail.com

\section{Juliane de Souza Scherer}

Mestre em Ciências da Saúde - Cardiologia (FUC-2012). Especialista em Administração dos Serviços de Enfermagem (IACHS-1999). Professora assistente do curso de Enfermagem e do Programa de Pósgraduação e Residência Multiprofissional da Universidade Feevale. Atualmente, é doutoranda do Programa de Pós-graduação em Patologia da UFCSPA. sjuliane@feevale.br 


\section{André Luis Machado Bueno}

Mestre em Enfermagem pela UFRGS (2010). Doutor em Enfermagem pela UFRGS (2017). Atualmente, é professor da Universidade Feevale, docente em disciplinas teóricas e práticas relacionadas ao cuidado do paciente adulto e idoso. Desenvolve atividades de pesquisa com ênfase em estudos epidemiológicos, ligadas às temáticas das causas externas, violência de gênero e sistemas de informação em saúde. andrebueno@feevale.br

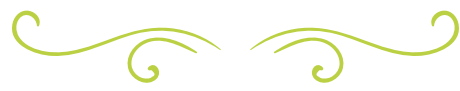

\section{RESUMO}

A Pneumonia Associada à Ventilação Mecânica (PAVM) tornou-se o evento adverso mais temível nas Unidades de Terapia Intensiva (UTI). Devido à importância e gravidade do tema, as instituições hospitalares têm aderido ao uso de bundles, que visam minimizar a incidência de PAV. Este trabalho teve como objetivo a elaboração de uma nova tecnologia para contribuir em melhorias na área da saúde. Por meio da criação de uma aplicação web, denominada SISPREV, foi construído um protótipo para realização da coleta de dados do bundle de prevenção de PAV. A pesquisa foi aprovada pelo Comitê de Ética em Pesquisa (CEP), sob o número 2.721.882. A aplicação web gera relatórios a partir dos dados coletados e dinamiza a monitorização de medidas preventivas de PAV em tempo real. Por meio do estudo, aplicado em 41 pacientes, durante um mês, foi possível verificar a agilidade na compilação dos dados no cenário atual, analisando a adesão da equipe às intervenções que envolvem a prevenção de danos. Os resultados possibilitam uma abordagem em tempo real dos dados gerados, permitindo o manejo de ações corretivas para adequação ao protocolo de prevenção, na busca por melhorias, promovendo segurança na assistência, redução de custos e mudanças de efeitos duradouros nos processos de trabalho.

Palavras-chave: Prevenção. Pneumonia Associada à Ventilação Mecânica. Unidade de Terapia Intensiva. Software.

\section{ABSTRACT}

Ventilator-associated Pneumonia (VAP) has become the most feared adverse event in Intensive Care Units (ICUs). Due to the importance and the seriousness of the theme, hospital institutions have adhered the bundles, which aim to minimize the incidence of VAP. This work aimed at the development of a new technology to contribute to improvements in the health area. Through the creation of a web application, called SISPREV, a prototype built to performing the data collection of the VAP prevention bundle. CEP approved this study under number $2,721,882$. The web application generates reports from the collected data and dynamizes the monitoring of preventive measures of VAP in real time. Through the study, applied to 41 patients, during one month, it was possible to verify the agility in the compilation of the data in the current scenario, analyzing the adhesion of the team to the interventions that involve the prevention of damages. The results allow an approach in real time, enabling to take correctives actions suitable to prevention protocol, searching improvements, furthering the security in the hospital assistance, cost cutting and changes of long lasting effects in the work processes.

Keywords: Prevention. Ventilator-associated Pneumonia. Intensive Care Units. Software. 


\section{INTRODUÇÃO}

A Pneumonia Associada à Ventilação Mecânica (PAVM) é definida como a infecção pulmonar no paciente em uso de ventilação mecânica invasiva, ao menos por 48 horas, ou após 24 horas da extubação do paciente. Dessa forma, a PAVM é uma grave infecção provocada por múltiplos fatores com consequente impacto na mortalidade, aumentando o tempo de permanência nas Unidades de Terapia Intensiva (UTI), o que reflete nos custos hospitalares (BRASIL, 2017a).

Devido à importância e gravidade do tema, as instituições hospitalares vêm buscando planos de prevenção e instituindo o uso de pacotes de medidas preventivas, tais como: manter cabeceira elevada (30-45\%), adequar diariamente o nível de sedação, aspirar secreção subglótica e realizar higiene oral com antisséptico a cada seis horas, entre outras, denominadas de bundles. Cabe ressaltar que os bundles são mais eficazes quando as medidas preconizadas são adotadas em conjunto e de maneira sistemática (SACHETTI et al., 2014; BRASIL, 2017b).

Para tanto, é recomendado realizar a vigilância da implantação e adesão das medidas preventivas, de maneira a monitorar os indicadores em busca de estratégias e melhorias para reduzir a PAVM. Além disso, a manutenção de rotinas de visitas multidisciplinares no intuito de corrigir inconformidades e identificar falhas nos processos assistenciais é fortemente indicada (BRASIL, 2017b). Nesse sentido, a busca por estratégias e medidas visando impactar a ocorrência desses eventos apresenta-se como desafio para as equipes multiprofissionais, sobretudo para as equipes de enfermagem, uma vez que o desenvolvimento de práticas adequadas, de forma sistematizada, é capaz de melhorar a qualidade do atendimento (FERREIRA et al., 2013; PULZI JÚNIOR; FERRAZ; LAPCHICK, 2015).

Atualmente, para monitorizar os processos assistenciais, são necessários profissionais para coletar os dados, digitá-los no sistema e que façam a sua compilação. Essas atividades normalmente não são realizadas em tempo real, tardando o manejo do paciente crítico.

A partir de pesquisas realizadas na internet e junto a empresas de desenvolvimento de software para a área da saúde, não foi encontrado um sistema capaz de controlar os bundles. Dessa forma, com o objetivo de melhorar os indicadores assistenciais relacionados à PAVM, foi criada a aplicação web SISPREV. Esse sistema considerou as dificuldades dos profissionais de saúde em monitorar a eficácia dos protocolos assistenciais em um hospital público no Sul do Brasil, em decorrência da necessidade de recursos humanos para compilar os dados e criar indicadores assistenciais.

Nos hospitais em que existe controle rigoroso de padrão preventivo relacionado a essa infecção, o número de casos tem apresentado redução nos últimos anos, indicando que as PAVM são complicações evitáveis, a partir da organização assistencial com origem na geração de dados locais (BRASIL, 2017b). Sendo assim, esta pesquisa teve como objetivo a elaboração de um sistema de formulário eletrônico, a fim de contribuir para melhorias na área da saúde, sistema esse que tem a capacidade de monitorar a adesão às medidas preventivas em tempo real.

\section{MÉTODO}

O presente estudo trata-se de uma pesquisa de avaliação do SISPREV, com uma abordagem quantitativa, descritiva e exploratória, com base em dados gerados por meio de um sistema web, desenvolvido pelo Grupo de Pesquisa em Computação Aplicada, em Novo Hamburgo, Brasil, em conjunto com o curso de Enfermagem. Esse grupo de pesquisa é liderado por professores das áreas de Ciência da Computação e Enfermagem e tem por objetivo a realização 
de pesquisas, desenvolvimento, avaliação e validação de ferramentas tecnológicas que apoiem o ensino na área da saúde.

O estudo foi realizado em um hospital público de ensino em Porto Alegre. A instituição possui 843 leitos no total. Destes, 59 são pertencentes à UTI, divididos em 14 leitos na Área Um, 16 leitos na Área Dois, 14 na Área Três e 15 leitos na Área Quatro. A população do presente estudo foi composta por 41 pacientes em uso de Ventilação Mecânica (VM), na UTI Área dois, sendo excluídos os que não estavam em ventilação mecânica no momento da aplicação do estudo. Foram totalizadas, durante o período de coleta, 268 avaliações.

Para avaliação do SISPREV, foram incluídos, nesta pesquisa, todos os pacientes em Ventilação Mecânica Invasiva internados na UTI Área Dois. Esta foi escolhida devido ao perfil do paciente assistido nessa área paciente clínico com estadia hospitalar mais prolongada em relação às demais áreas, proporcionando maior precisão e regularidade na coleta de dados. Foram excluídos aqueles que não contemplaram os critérios de inclusão, assim como aqueles que não estavam fazendo uso de Ventilação Mecânica Invasiva. O período de coleta dos dados da validação foi de 30 dias, de 28 de agosto a 26 de setembro de 2018 .

O protótipo de um sistema de formulários, SISPREV, foi desenvolvido e tem disponibilidade de acesso a partir de qualquer computador ou dispositivo móvel que possua acesso à internet. A aplicação foi desenvolvida nas linguagens PHP (Php Hypertext Preprocessor), SQL (Structured Query Language), HTML5 (Hypertext Markup Language), CSS (Cascading Style Sheets) e Javascript. A linguagem PHP foi responsável por realizar a interação entre 0 cliente (navegador web) e o servidor, utilizando o SQL para a manipulação e consulta de dados no SGBD (Sistema Gerenciador de Banco de Dados) MySQL.

As linguagens HTML5, Javascript e CSS foram utilizadas para o desenvolvimento de componentes visuais, em que os usuários interagem no navegador. Esse sistema está hospedado em um servidor e pode ser acessado por meio do link: http://ceted.feevale. br/protocolos. Os dados, portanto, foram enviados para o SGBD MySOL, responsável por armazenar os registros coletados, e analisados por profissionais da saúde por meio dos relatórios gerados pelo SISPREV. Os profissionais de saúde autorizados podem acessar a aplicação utilizando e-mail e senha previamente cadastrados pelo administrador da aplicação.

A aplicação está dividida em duas seções: um menu à esquerda e uma seção por meio da qual o usuário interage com os dados. No menu à esquerda, o usuário pode escoIher entre as opções: Home, Formulários, Relatórios e Pacientes. No item Home há somente uma breve descrição a respeito do projeto; o item Formulários permite ao usuário interagir com os bundles cadastrados, nesse momento somente o bundle para prevenção da PAVM está disponível. Os Relatórios permitem ao usuário verificar informações estatísticas relacionadas à aplicação das medidas de prevenção nos pacientes. O último item, Pacientes, possibilita a criação e edição dos dados referentes aos pacientes internados.

Dessa forma, a aplicação permite que - usuário faça as interações necessárias e registre os dados em um SGBD MySOL. Este, por sua vez, salva os dados em uma série de tabelas em que ficam armazenados os usuários da aplicação, os pacientes, os formulários cadastrados, as perguntas dos formulários, as respostas das intervenções dos usuários para cada formulário/paciente, entre outras informações.

Por meio da criação de uma aplicação web, denominada SISPREV, foi construído um protótipo para realização da coleta de dados do bundle de prevenção de PAVM. Após coletados, os dados foram enviados para um servidor, possibilitando a análise dessas informações por profissionais da área da saúde em tempo real. O sistema 
possibilita gerar relatórios a partir dos dados coletados, sem a necessidade de profissionais específicos para digitá-los, e visa dinamizar a aplicação de medidas para prevenção de PAVM.

Vale ressaltar que a aplicação registra os dados dos pacientes sem a identificação do indivíduo. Também é possível obter controle das avaliações realizadas no paciente, durante sua estadia no hospital, possibilitando o registro de informações do formulário de avaliação, as patologias do paciente, datas de entrada e saída e o nome do responsável pela avaliação.

Cada bundle corresponde a um formulário no SISPREV. O formulário, portanto, dá acesso a uma série de perguntas, conforme o protocolo correspondente. Esse protótipo permite que outros bundles de prevenção sejam anexados à plataforma, auxiliando os profissionais de saúde no acompanhamento dos processos assistenciais que necessitem de monitoramento de ações diárias.
A Figura 1 ilustra o bundle de prevenção de PAVM, exibindo algumas perguntas.

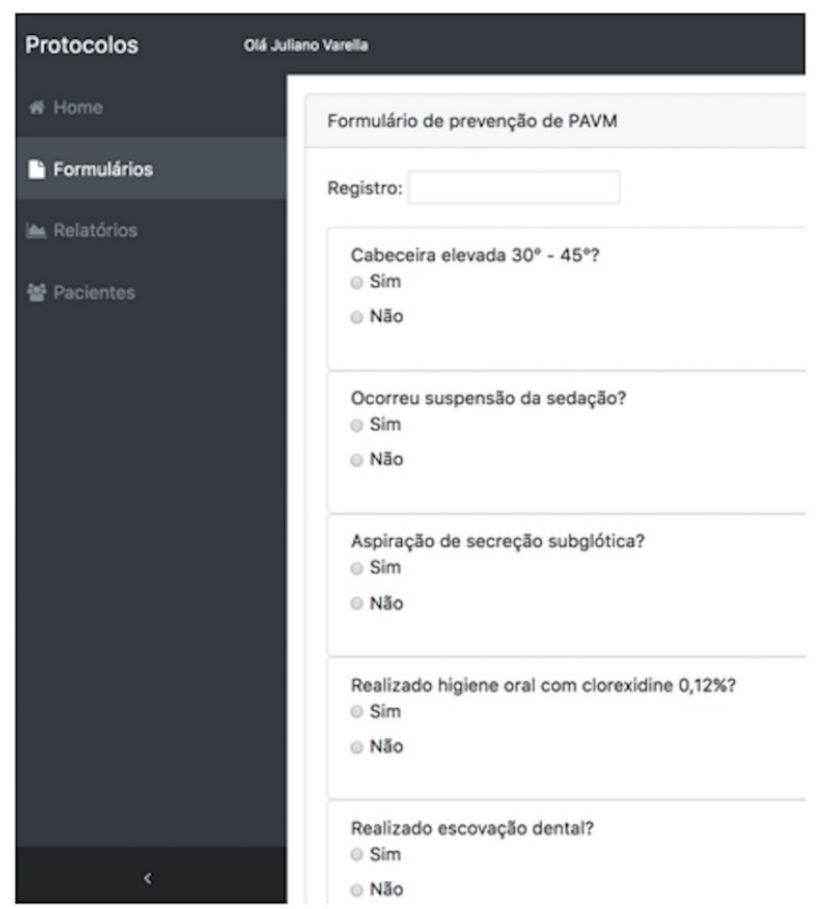

Figura 1 - Formulário com as perguntas associadas à prevenção de PAVM.

Fonte: SISPREV.

O SISPREV permite adicionar novos pacientes, assim como dá acesso à lista de pacientes já cadastrados. Ao clicar no botão "Abrir", do paciente desejado, é possível cadastrar e editar as informações pessoais dele, bem como adicionar e editar avaliações realizadas sobre o paciente. A Figura 2 exibe essas interações realizadas sobre um paciente.
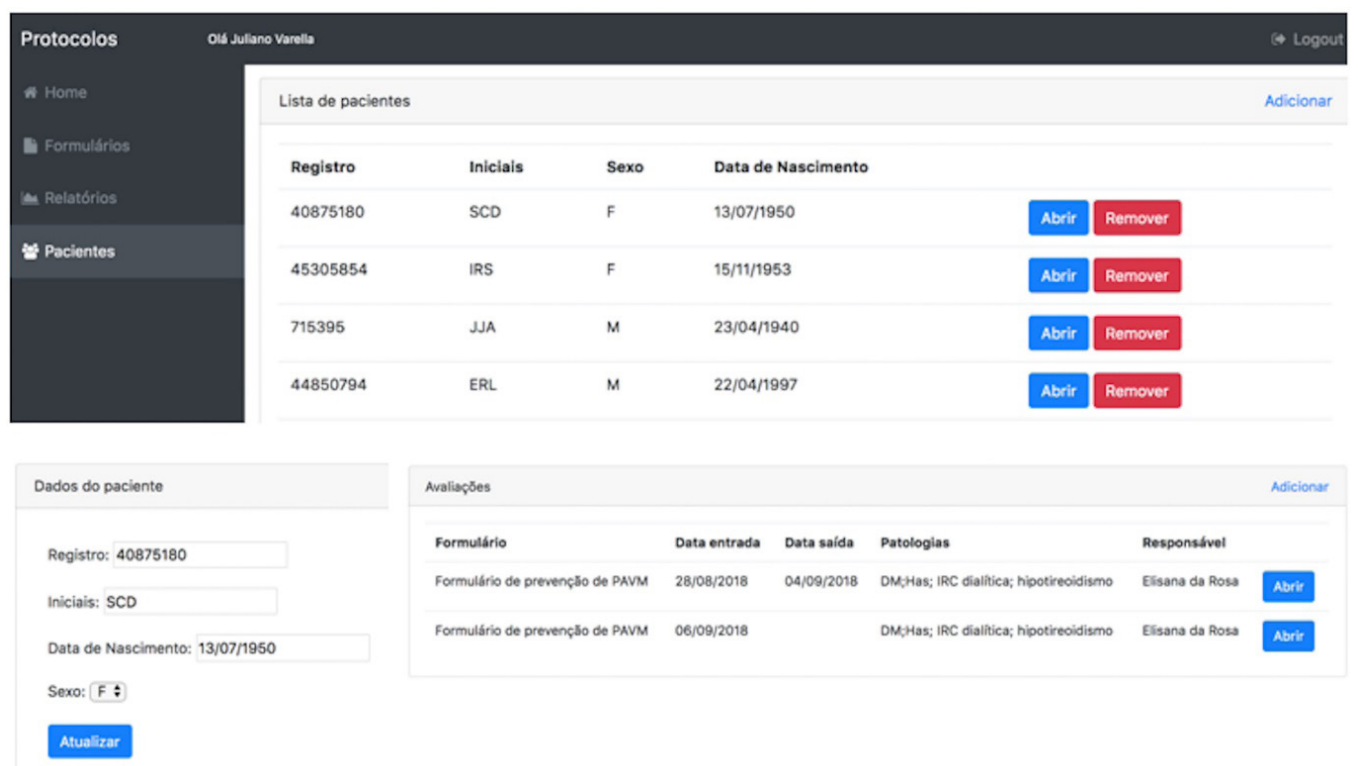

Figura 2 - Lista dos pacientes registrados, informações de um paciente específico e duas avaliações deste paciente. Fonte: SISPREV. 
Dessa forma, a aplicação permite aferir o paciente em diferentes períodos do dia, sendo possível obter um diagnóstico mais preciso dos resultados das avaliações. Estas possuem o registro de data e hora, para se saber os períodos em que o paciente foi avaliado. Para cada avaliação, é possível cadastrar eventos para fins de registro ou relatórios.

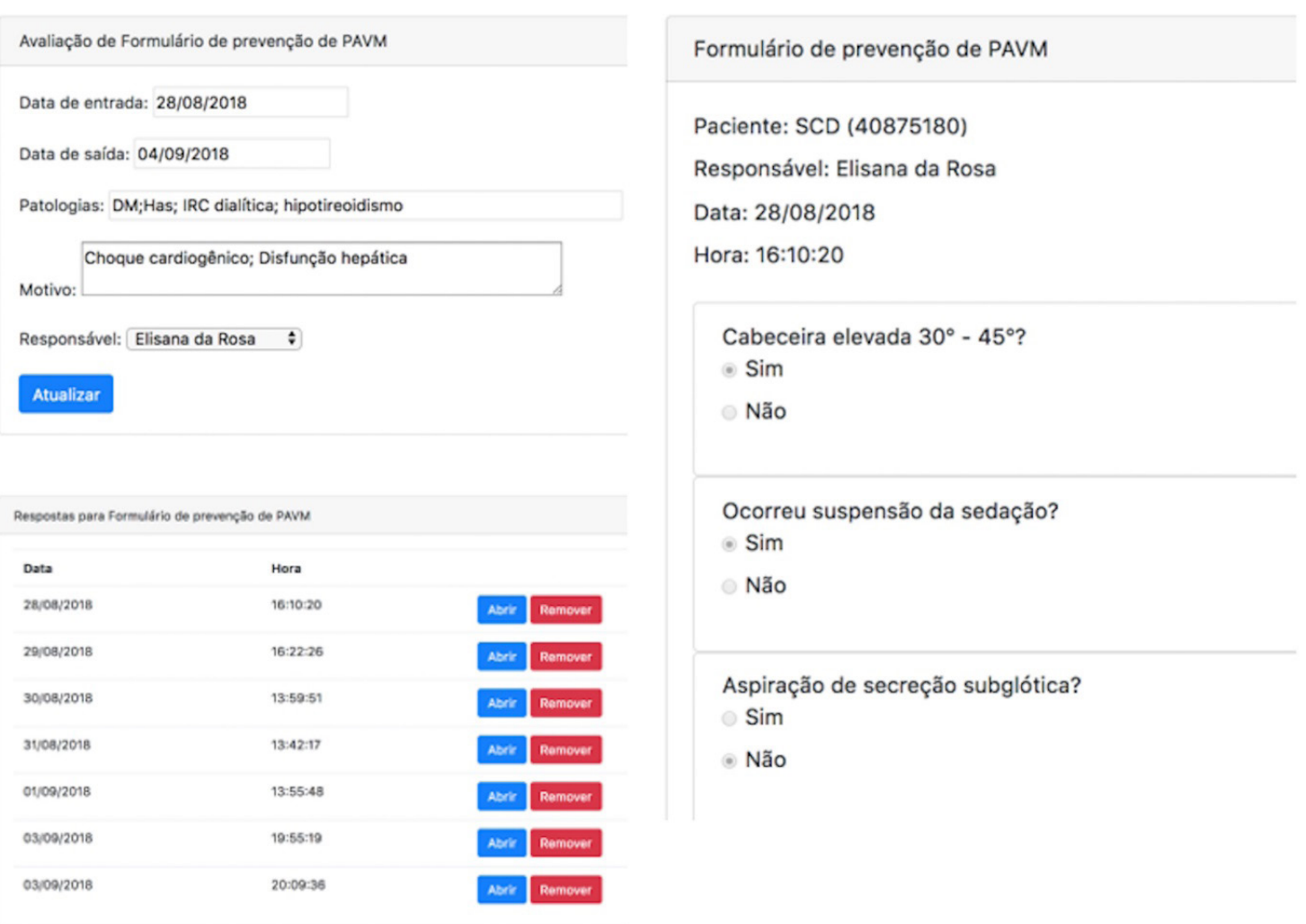

A Figura 3 ilustra uma avaliação realizada para um paciente específico e as diversas respostas preenchidas do formulário em momentos diferentes. Ressalta-se que o SISPREV também pode ser manipulado por quem presta a assistência direta ao paciente, eliminando a necessidade de outros profissionais administrativos, tornando o processo mais rápido e ágil para a assistência.

Figura 3 - Avaliação de um paciente no formulário de PAVM com respostas em diversos momentos. Fonte: SISPREV.

A partir dos relatórios, é possível visualizar os dados coletados dos pacientes, tanto de forma individual como em grupo, aplicando o bundle de prevenção de PAVM. Esses relatórios contêm as porcentagens das respostas informadas para cada pergunta do formulário.

A presente pesquisa considera toda regulação de ética em pesquisa, prevista na Resolução 466/2012 do Conselho Nacional de Saúde (CNS). Para isso, a resolução engloba referenciais de bioética, como autonomia, beneficência e a não maleficência, justiça, equidade, entre outros, respeitando os direitos e deveres dos indivíduos que compõem a pesquisa (BRASIL, 2012). O projeto foi para análise do Comitê de Ética em Pesquisa (CEP), sendo os dados coletados somente após aprovação, sob o número do parecer 2.721.882.

Depois de autorizada a pesquisa, a pesquisadora apresentou-se na instituição diariamente, durante trinta dias, para a coleta das informações, fazendo registros de dados no SISPREV. $O$ instrumento de pesquisa é composto por dez questões, que agrupadas formam um bundle de prevenção de PAVM e foram disponibilizadas por meio de um formulário na aplicação web. 


\section{RESULTADOS E DISCUSSÃO}

Os resultados da aplicação do SISPREV em uma UTI de um Hospital de Escola do Sul do Brasil, 100\% SUS, com a coleta de dados executada por apenas uma pessoa, demonstraram uma adesão de $91 \%$ ao bundle aplicado para prevenção de PAVM. Entre as dez medidas aplicadas e avaliadas diariamente, sete obtiveram adesão superior a 90\%, demonstrando o envolvimento da equipe multidisciplinar na observância das medidas.

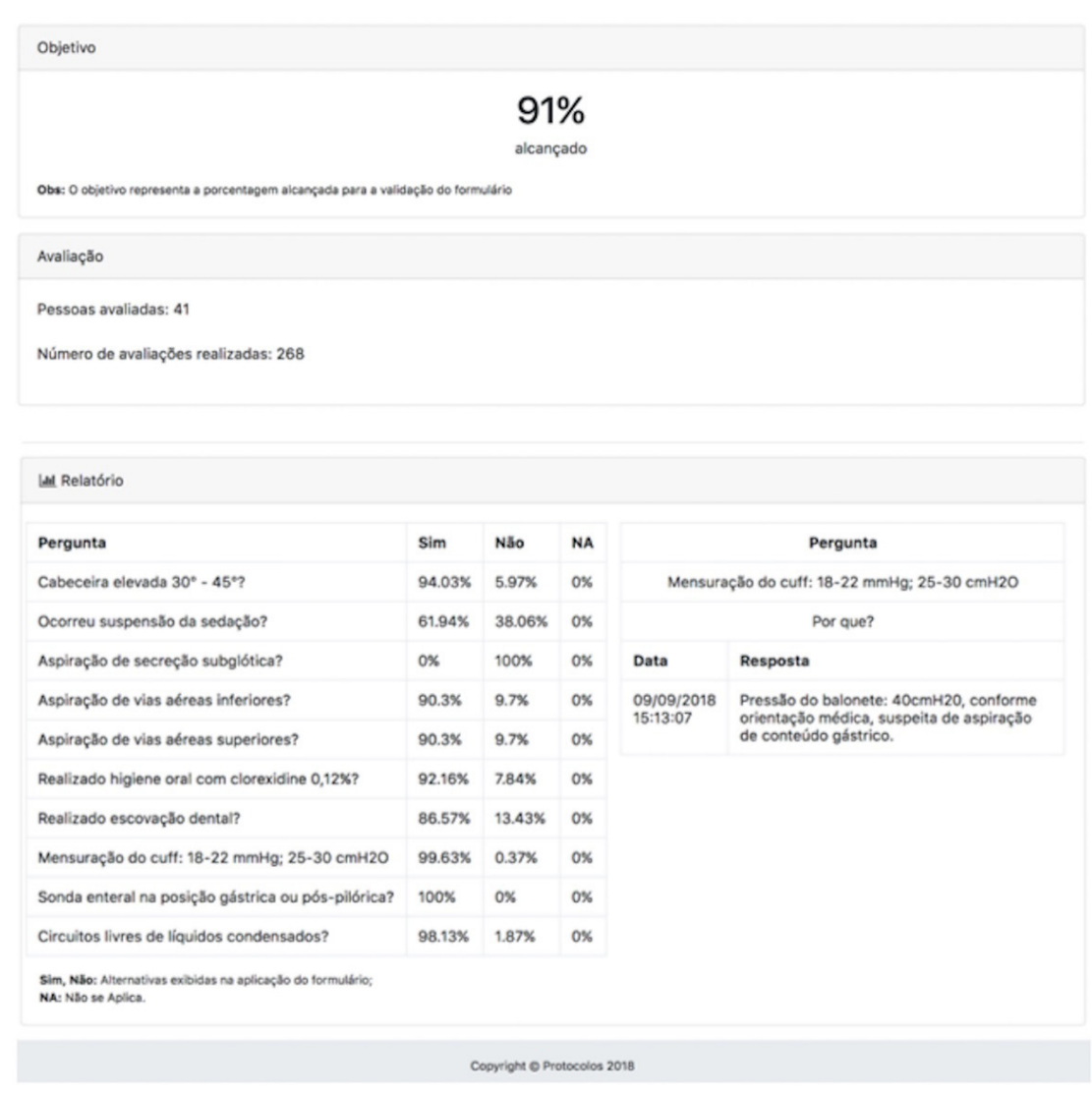

Figura 4 - Relatório com as estatísticas das avaliações realizadas. Fonte: SISPREV.

Na Figura 4, pode-se constatar o predomínio de $100 \%$ de adesão em relação à manutenção do posicionamento da sonda enteral em região gástrica ou pós-pilórica. Em seguida, em relação à medida de mensuração e manutenção do cuff, mantendo-a com uma pressão de 18-22 mmHg ou 25-30 $\mathrm{cmH} 2 \mathrm{O}$, observou-se uma adesão da equipe de $99,63 \%$. A manutenção da cabeceira elevada apresentou resultados de 94,03\% de adesão, um percentual satisfatório, demonstrando comprometimento da equipe frente a essa medida preventiva.

A medida de prevenção de aspiração subglótica apresentou-se 100\% negativa.
Esse fato justifica-se pela atual inexistência do dispositivo necessário para o procedimento na instituição onde o estudo foi realizado. Outra medida registrada foi à adesão à suspensão de sedação diária com 38,06\% de negativa.

A Figura 4, resultante do relatório do SISPREV, torna perceptível a adesão desejável no cuidado com a realização da higiene oral com clorexidine $0,12 \%$, com aplicação em $92,16 \%$ dos casos avaliados. Em contrapartida, a escovação dental obteve uma adesão abaixo do esperado, com somente $86,57 \%$ de conformidade. 
A redução das taxas de PAVM depende do empenho e da disposição da equipe de enfermagem e dos demais profissionais. As intervenções aplicadas juntamente com ações colaborativas de cuidado são base para precaução de todo o risco que esse paciente possa ter e para diminuição dos números expressivos dessa infecção (ALMEIDA, 2015; WAGNER et al., 2015).

Diversos aspectos e atitudes precisam ser monitorados para garantir a segurança do paciente no ambiente de cuidados intensivos. $O$ plano para minimizar os riscos de aspiração em pacientes em VM e otimizar o fornecimento da nutrição enteral é o posicionamento da sonda para administrar a dieta. A recomendação é considerar duas vias, gástrica e/ou pós-pilórica, preferindo o posicionamento pós-pilórico para pacientes com intolerância e/ou contraindicação gástrica (BARBAS et al., 2015).

O cuff constitui-se em um balonete que apresenta como função primária o fechamento das vias aéreas inferiores, promovendo uma ventilação apropriada ao paciente em VM e prevenindo a aspiração de secreção subglótica (LEAL et al., 2017). O cuidado com a pressão correta do cuff é essencial em pacientes em uso de ventilação mecânica, pois a pressão acima do recomendado pode comprometer a microcirculação da mucosa traqueal e causar lesões isquêmicas. Porém, se a pressão for menor que o desejado, a ventilação artificial oferecida com pressão positiva não será adequada, além de favorecer a passagem de secreções acumuladas acima do cuffpor entre o tubo e a traqueia. É preconizado, portanto, que a pressão do cuff permaneça entre 18 a $22 \mathrm{mmHg}$ ou 25 a $30 \mathrm{~cm} \mathrm{H}_{2} \mathrm{O}$, sempre evitando pressões maiores que 22 $\mathrm{mm} \mathrm{Hg}$ ou $30 \mathrm{~cm} \mathrm{H}_{2} \mathrm{O}$ (CARDOSO; BIZANI, 2015; BRASIL, 2017b).

O paciente em uso de ventilação mecânica está suscetível a infecções, visto que o tubo endotraqueal debilita a barreira natural entre a orofaringe e a traqueia, deixando os fatores de proteção do sistema respiratório diminuídos. A sedação, necessária para conforto do paciente intubado, suprime o reflexo de tosse, e o acúmulo de secreções acima do balonete do tubo favorecem a aspiração dessas secreções para o trato respiratório inferior (SILVA; NASCIMENTO; SALLES, 2012).

A remoção mecânica de secreções, por meio da aspiração endotraqueal, visa manter as vias aéreas permeáveis e a prevenção de doenças. A diminuição do acúmulo de secreções subglóticas é imprescindível para manter as vias aéreas pérvias e para reduzir o risco de consolidação e atelectasias (SILVA et al., 2014).

No que diz respeito ao uso do sistema fechado de aspiração, estudos afirmam que ele reduz os riscos de atelectasias, hipoxemia, arritmias, contaminação, acidentes ocupacionais ao trabalhador e deve ser empregado principalmente em situações de PEEP (Pressão Positiva Expiratória Final) elevada, como em casos de lesão pulmonar aguda (RIBEIRO; ANJOS; OLIVEIRA, 2016).

Pacientes que necessitam de ventilação mecânica precisam de uso sistemático de sedativos para conforto e melhora no padrão ventilatório. Entretanto, a sedação profunda acaba impedindo o desmame precoce, elevando o risco de pneumonia associada à VM (SILVA; NASCIMENTO; SALLES, 2013).

Considerando a extubação, é importante a avaliação do paciente. Essa análise deve ser realizada diariamente com a interrupção da sedação. Nesse cenário, cabe à equipe de enfermagem atentar para as reações do paciente, comunicando a equipe médica, registrando e realizando o monitoramento contínuo para evitar extubação acidental e queda do paciente (FERREIRA et al., 2013). A relação entre o perío do de VM e os índices de PAVM propõe a realização de um trabatho coeso entre os profissionais da equipe que assistem o paciente grave, com o intuito de antecipar extubação e evitar sedações indevidas (CARDOSO; BIZANI, 2015).

A higiene da cavidade oral requer um cuidado especial pela enfermagem, 
principalmente em pacientes de cuidados intensivos. Essa preocupação se explica pelo constante acúmulo de secreções na orofaringe e pelo fato de os pacientes serem incapazes de eliminá-los pela perda do reflexo de tosse e pelo sistema mucociliar deficiente. Sendo assim, a colonização da cavidade oral por microrganismos gram-negativos multirresistentes passa a ser uma importante via para a ocorrência de PAVM (NEPOMUCENO et al., 2014).

No Brasil, a Sociedade Brasileira de Pneumologia e Tisiologia preconiza a descontaminação da cavidade bucal com clorexidina ou com clorexidina associada à colistina, na prevenção de pneumonia associada à VM. O uso tópico de clorexidina na higiene oral de pacientes em uso de ventilação mecânica parece reduzir a colonização da cavidade bucal, podendo diminuir as taxas da PAVM. Desse modo, fica evidente que esse procedimento é seguro e bem tolerável, já que não foram demonstrados efeitos colaterais em nenhum estudo, sendo considerada uma medida de baixo custo (MICHELS et al., 2013).

A ANVISA preconiza o uso da clorexidina aquosa 0,12\%, que tem ação bactericida (BRASIL, 2017b). No entanto, a remoção de placa bacteriana deve ser realizada por meio mecânico, utilizando dispositivos que cheguem às superfícies dentárias e aos espaços interdentários, como escovas dentárias descartáveis de cerdas macias e fios flexíveis associados à clorexidina em solução aquosa a 0,12\%.

A utilização do SISPREV, como demonstrada anteriormente, promoveu a verificação de aspectos essenciais para a segurança do paciente no ambiente de cuidados intensivos. $\bigcirc$ sistema proporciona agilizar $\mathrm{O}$ processamento de dados, substituindo o que antes era realizado de forma manual, permitindo um panorama, em tempo real, das práticas de prevenção aplicadas pela equipe assistencial. A eficiência do SISPREV permite ao gestor detectar a falha na assistência, traçar metas e aplicar medidas de ações corretivas em tempo oportuno, beneficiando a evolução do estado clínico do paciente gravemente enfermo.

Cabe ressaltar que o trabalho em equipe do grupo de Computação Aplicada, no qual interagem profissionais de ciência da computação e enfermeiros, proporcionou o surgimento de melhorias assistenciais, integrando conhecimentos distintos, estimulando a busca por aprimoramento, focando na qualidade do processo assistencial, evitando danos aos pacientes críticos e, consequentemente, diminuindo o tempo de permanência na unidade de tratamento intensivo.

Segundo Barra e Dal Sasso (2010), existem muitos estudos apontando que as Tecnologias da Informação e Comunicação (TIC) melhoram o cuidado direto ao paciente, assim como reduzem o tempo de documentação e registro das informações relacionadas ao cuidado. Cada tecnologia aplicada ao cuidado aumenta a complexidade do trabalho da enfermagem, embora apresente redução da carga de trabalho, melhorando a qualidade do cuidado e minimizando os eventos adversos. Afirma, ainda, que o volume de informações na UTI é enorme e que essas informações são heterogêneas, complexas e, muitas vezes, pouco organizadas.

Informações são cruciais no processo do cuidado e o acesso a elas corrobora evidências para sustentar as ações da enfermagem na assistência ao paciente grave. Logo, há um grande encadeamento entre acesso à informação, resultados e a segurança do paciente no ambiente hospitalar (BARRA; DAL SASSO, 2010; GOMES et al., 2017).

\section{CONCLUSÃO}

No decorrer da pesquisa, podemos verificar a relevância de uma tecnologia que garanta a compilação dos dados de prevenção de PAVM em tempo real, visto que a segurança do paciente no âmbito hospitalar 
reflete a qualidade da assistência prestada por uma instituição.

O comportamento da equipe de enfermagem é fator determinante frente à PAVM, proporcionando cuidados permanentes ao paciente. Para isso, deve-se procurar o aprimoramento frequente, a fim de corrigir falhas e melhorar o desempenho das pessoas em relação ao processo de cuidado, na busca por resultados otimizados e adaptados às diferentes realidades assistenciais.

Por meio deste estudo, com a utilização do SISPREV, foi possível perceber, ainda, a redução de custos com recursos humanos para a coleta de dados, visto que antes era necessária a contratação de um profissional para realizar esse tipo de avaliação. O custo também se reduz devido à ausência de trabalho manual na compilação e geração de relatórios, agora consolidados pelo SISPREV.

Nesse sentido, o SISPREV tem capacidade de sinalizar falhas nos processos assistenciais, identificando-as na aplicação de medidas preventivas imediatamente. Isso possibilita ao gestor atuar na identificação das fragilidades em tais processos, a fim de promover a capacitação de recursos humanos com base em dados locais, fortalecendo, dessa forma, ações educativas em tempo e espaço próprios.

Por fim, este estudo verificou a agilidade e a fácil utilização do sistema, favorecendo a compilação e análise dos dados, além da adesão da equipe às intervenções que envolvem a prevenção de PAVM. Os resultados possibilitam uma abordagem em tempo real dos dados gerados, permitindo o manejo de ações corretivas para adequação ao protocolo de prevenção, promovendo segurança na assistência e mudanças de práticas com efeitos duradouros e impactantes nos processos de trabalho. Como trabalhos futuros, pretende-se aplicar novamente o bundle de PAVM em outras oportunidades, bem como incluir outros bundles no sistema SISPREV. 


\section{REFERÊNCIAS}

ALMEIDA, K. M. V. Adesão às medidas de prevenção para pneumonia associada à ventilação mecânica. Revista de

Enfermagem UFSM, v. 5, n. 2, p. 247-256, 2015.

BARBAS, C. S. V. et al. Recomendações brasileiras de ventilação mecânica 2013: Parte I. Revista Brasileira de Terapia Intensiva, v. 26, n. 2, p. 89-121, 2015.

BARRA, D. C. C.; DAL SASSO, G. T. M. Tecnologia móvel à beira do leito: processo de enfermagem informatizado em terapia intensiva a partir da Cipe 1.0®. Texto Contexto Enferm, Florianópolis, v. 19, n. 1, p. 54-63, jan./mar. 2010.

BRASIL. Agência Nacional de Vigilância Sanitária. Critérios Diagnósticos de Infecções Relacionadas à Assistência à Saúde. Brasília: Anvisa, 2017a.

BRASIL. Agência Nacional de Vigilância Sanitária. Medidas de Prevenção de Infecção Relacionada à Assistência à Saúde. 2. ed. Brasília: Anvisa, 2017b. Disponível em: https://www20.anvisa.gov. br/segurancadopaciente/index.php/publicacoes/item/caderno-5. Acesso em: 2 out. 2019.

\section{BRASIL. Resolução - RDC, N 15, de 15 de março de 2012.}

Dispõe sobre requisitos de boas práticas para o processamento de produtos para saúde e dá outras providências. 2012. Disponível em: http://bvsms.saude.gov.br/bvs/saudelegis/anvisa/2012/ rdc0015_15_03_2012.html. Acesso em: 2 out. 2019.

CARDOSO, M. E. V.; BIZANI, D. Aplicação de bundle de prevenção de pneumonia associada à ventilação mecânica em centro de terapia intensiva adulto: um relato de experiência. Revista

Saúde e Desenvolvimento Humano, v. 3, n. 2, p. 137-146, 2015. Disponível em: https://revistas.unilasalle.edu.br/index.php/saude_ desenvolvimento/article/view/2317-8582.15.11. Acesso em: 2 out. 2019.

FERREIRA, A. B. et al. Práticas de Enfermagem que podem minimizar a ocorrência de pneumonia associada à ventilação mecânica invasiva em Unidade de Terapia Intensiva. Periódico Científico do Núcleo de Biociências Centro Universitário Metodista, v. 3, n. 5, p. 15-31, 2013.

GOMES, A. T. L. et al. Tecnologias aplicadas à segurança do paciente: uma revisão bibliométrica. Revista de Enfermagem do 
Centro-Oeste Mineiro, v. 7, e1473, 2017. Disponível em: https://doi. org/10.19175/recom.v7i0.1473. Acesso em: 2 out. 2019.

LEAL, G. A. et al. Cuidados de enfermagem para prevenção da pneumonia associada à ventilação mecânica em Unidades de Terapia Intensiva: uma revisão literária. Cadernos de Graduação, Aracaju, v. 4, n. 1, p. 95-108, 2017. Disponível em: https://periodicos. set.edu.br/index.php/cadernobiologicas/article/view/3657. Acesso em: 2 out. 2019.

MICHELS, M. A. et al. Auditoria em unidade de terapia intensiva: vigilância de procedimentos invasivos. Revista de Epidemiologia e Controle de Infecção, v. 3, n. 1, p. 12-16, 2013. Disponível em: https://online.unisc.br/seer/index.php/epidemiologia/article/ view/2741. Acesso em: 2 out. 2019.

NEPOMUCENO, R. M. et al. Fatores de risco modificáveis para pneumonia associada à ventilação mecânica em Terapia Intensiva.

Revista de Epidemiologia e Controle de Infecção, v. 4, n. 1, p. 23-27, 2014. Disponível em: https://online.unisc.br/seer/index.php/ epidemiologia/article/view/3933. Acesso em: 2 out. 2019.

PULZI JÚNIOR, S. A.; FERRAZ, R. R. N.; LAPCHICK, M. S. Pneumonia associada à ventilação mecânica como indicador de qualidade e segurança em saúde. Rev Med Minas Gerais, v. 25, n. 4, p. 517-522, 2015. Disponível em: http://bases. bireme.br/cgi-bin/wxislind.exe/iah/online/?lsisScript=iah/iah. xis\&src $=$ google\&base $=$ LILACS\&lang $=p \&$ nextAction $=$ Ink\&exprSearch=774699\&indexSearch=ID. Acesso em: 2 out. 2019.

RIBEIRO, K. R. A.; ANJOS, E. G.; OLIVEIRA, E. M. Enfermagem em ventilação mecânica: cuidados na prevenção de pneumonia. Revista Recien, São Paulo, v. 6, n. 16, p. 57-71, 2016.

SACHETTI, A. et al. Adesão às medidas de um bundle para prevenção de pneumonia associada à ventilação mecânica.

Revista Brasileira de Terapia Intensiva, v. 26, n. 4, p. 355-359, 2014. Disponível em: http://www.scielo.br/scielo.php?pid=S0103507X2014000400355\&script=sci_abstract\&tlng=pt. Acesso em: 2 out. 2019.

SILVA, S. G. et al. Avaliação de um bundle de prevenção da pneumonia associada à ventilação mecânica em Unidade de Terapia Intensiva. Texto Contexto Enfermagem, Florianópolis, v. 23, n. 3, p. 744-750, 2014. 
SILVA, S. G.; NASCIMENTO, E. R. P.; SALLES, R. K. Bundle de prevenção da pneumonia associada à ventilação mecânica: uma construção coletiva. Texto Contexto Enfermagem, Florianópolis, v. 21, n. 4, p. 837-844, 2012. Disponível em: http://www.scielo.br/scielo. php?script=sci_arttext\&pid=S0104-07072012000400014. Acesso em: 2 out. 2019.

SILVA, S. G.; NASCIMENTO, E. R. P.; SALLES, R. K. Pneumonia associada à ventilação mecânica: discursos de profissionais acerca da prevenção. Escola Anna Nery Revista de Enfermagem, v. 18, n. 2, p. 290-295, 2013. Disponível em: http://www.scielo.br/scielo. php?script=sci_arttext\&pid=S1414-81452014000200290. Acesso em: 2 out. 2019.

WAGNER, B. V. et al. O conhecimento do enfermeiro acerca das intervenções destinadas à prevenção da pneumonia associada à ventilação mecânica. Revista de Enfermagem UFPE, Recife, v. 9, n. 5, p. 7902-7909, 2015. Disponível em: https://periodicos.ufpe.br/ revistas/revistaenfermagem/article/download/10540/11448. Acesso em: 2 out. 2019. 